\section{Longitudinal high-resolution imaging through a flexible intravital imaging window}

\author{
Guillaume Jacquemin ${ }^{1 *}$, Maria Benavente-Diaz ${ }^{2,3,4}$, Samir Djaber ${ }^{1}$, \\ Aurélien Bore $^{1,5}$, Virginie Dangles-Marie ${ }^{6,7}$, Didier Surdez ${ }^{8 \dagger}$, Shahragim Tajbakhsh ${ }^{2,3}$, \\ Silvia Fre ${ }^{1 *}$, Bethan Lloyd-Lewis ${ }^{1,9 *}$
}

\begin{abstract}
Intravital microscopy (IVM) is a powerful technique that enables imaging of internal tissues at (sub)cellular resolutions in living animals. Here, we present a silicone-based imaging window consisting of a fully flexible, sutureless design that is ideally suited for long-term, longitudinal IVM of growing tissues and tumors. Crucially, we show that this window, without any customization, is suitable for numerous anatomical locations in mice using a rapid and standardized implantation procedure. This low-cost device represents a substantial technological and performance advance that facilitates intravital imaging in diverse contexts in higher organisms, opening previously unattainable avenues for in vivo imaging of soft and fragile tissues.
\end{abstract}

Copyright @ 2021

The Authors, some rights reserved; exclusive licensee American Association for the Advancement of Science. No claim to original U.S. Government Works. Distributed under a Creative Commons Attribution License 4.0 (CC BY)

\section{INTRODUCTION}

Bioimaging across multiple scales is a universal and mainstay tool in life science research. Technological advances in live and deep tissue imaging methodologies, including intravital microscopy (IVM), now enable the real-time microscopic imaging of individual cells within intact tissues in near physiological conditions (1-3). This powerful approach is increasingly leveraged in experimental and preclinical studies to reveal novel insights into the dynamic cellular mechanisms underlying disease development and response to therapy $(4,5)$. To facilitate repeated IVM over prolonged time periods in the same living animal, multiple imaging windows have been designed to provide optical access to internal tissues (6), including the brain $(7)$, skin $(8,9)$, lung $(10)$, mammary gland $(6,11,12)$, abdominal organs (13-15), femur (16), and embryos (17). Typically, these models consist of a glass coverslip inserted in a titanium (or more rarely plastic) frame and rely on sutures and/or glue to fix the window in place. Although powerful, this implantation methodcombined with the material composition and rigidity of conventional windows - is poorly suited to dynamic or rapidly growing soft tissues or tumors, often requiring customization to meet tissueor study-specific needs. The inherent rigidity of glass also limits its ability to adequately cover anatomical locations that exhibit curvature or joints. Collectively, these issues can cause animal distress, skin and tissue degradation, inflammation, fibrosis, and window detachment, ultimately leading to experimental failures. Moreover, the reliance of all conventional windows on suturing (and occasionally

\footnotetext{
${ }^{1}$ Institut Curie, Laboratory of Genetics and Developmental Biology, PSL Research University, INSERM U934, CNRS UMR3215, F-75248 Paris Cedex 05, France. ${ }^{2}$ Stem Cells \& Development Unit, Institut Pasteur, 25 rue du Dr. Roux, 75015 Paris, France. ${ }^{3}$ UMR CNRS 3738, Institut Pasteur, Paris, France. ${ }^{4}$ Sorbonne Universités, Complexité du Vivant, F-75005, Paris, France. ${ }^{5}$ CRISPR'it, Platform for Genetic Screens, Institut Curie, PSL Research University, INSERM U934, CNRS UMR3215, F-75248 Paris Cedex 05, France. ${ }^{6}$ Faculty of Pharmacy, Université Paris Descartes, Paris, France. ${ }^{7}$ In vivo Experiment Platform, PSL Research University, 75005 Paris, France. ${ }^{8}$ INSERM U830, Équipe Labellisée LNCC, Diversity and Plasticity of Childhood Tumors Lab, PSL Research University, SIREDO Oncology Centre, Institut Curie Research Centre, Paris, France. ' 9 chool of Cellular and Molecular Medicine, University of Bristol, Biomedical Sciences Building, Bristol, BS8 1TD, UK.

*Corresponding author. Email: guillaume.jacquemin@curie.fr (G.J.); silvia.fre@curie.fr (S.F.); bethan.Iloyd-lewis@bristol.ac.uk (B.L.-L.)

†Present address: Balgrist University Hospital, University of Zürich, 8008 Zürich, Switzerland.
}

glue) renders their implantation complicated and time-consuming. Finally, the absence of standardized and affordable imaging windows forces laboratories to make and reuse their own models, further precluding the universal and consistent application of IVM.

To address these limitations, here we developed a flexible and sutureless polydimethylsiloxane (PDMS)-based intravital imaging window dedicated to efficient, long-term maintenance at all body sites and improved animal welfare. While a few studies have reported the use of fixed-skull silicone membranes for brain IVM (18-20), the application of PDMS for soft tissue imaging remains unexplored, being limited to window designs that maintain the use of glass coverslips and sutures (21). Hence, these models provide no benefit over conventional rigid windows. By contrast, our design encompasses a flexible, seamlessly joined PDMS window that ensures a sealed barrier between the animal's internal tissues and the external environment in a suture-free manner. By thorough characterization of its optical properties, we validate the utility of the PDMS membrane as an optical material for deep tissue IVM. Alongside, we demonstrate the compatibility of the flexible window with intravital imaging at dynamic body locations and in conditions of extreme tissue growth, contexts that are less accessible to existing rigid windows. In turn, our engineered device promises to open new perspectives for in vivo imaging of soft and fragile tissues in higher vertebrates.

\section{RESULTS}

\section{PDMS-based intravital imaging window design}

To address the restraints of current rigid models, we designed a flexible intravital imaging window made entirely of PDMS (Fig. 1, A and B). PDMS is a light, biocompatible, chemically inert, and optically clear material that is commonly used in medical or microfluidic devices. It can be easily and reproducibly cast into any desired shape, size, thickness, and rigidity by varying mold footprints, silicone composition, and surface treatments. Collectively, these properties make PDMS an optimal material for devices demanding specifications for biocompatibility, versatility, and optical performance. Our PDMS-based intravital imaging window comprises a supple and lightweight frame surrounding a thinner $(145 \pm 10 \mu \mathrm{m})$ area for imaging (Fig. 1B). A priority for the design of a universally 

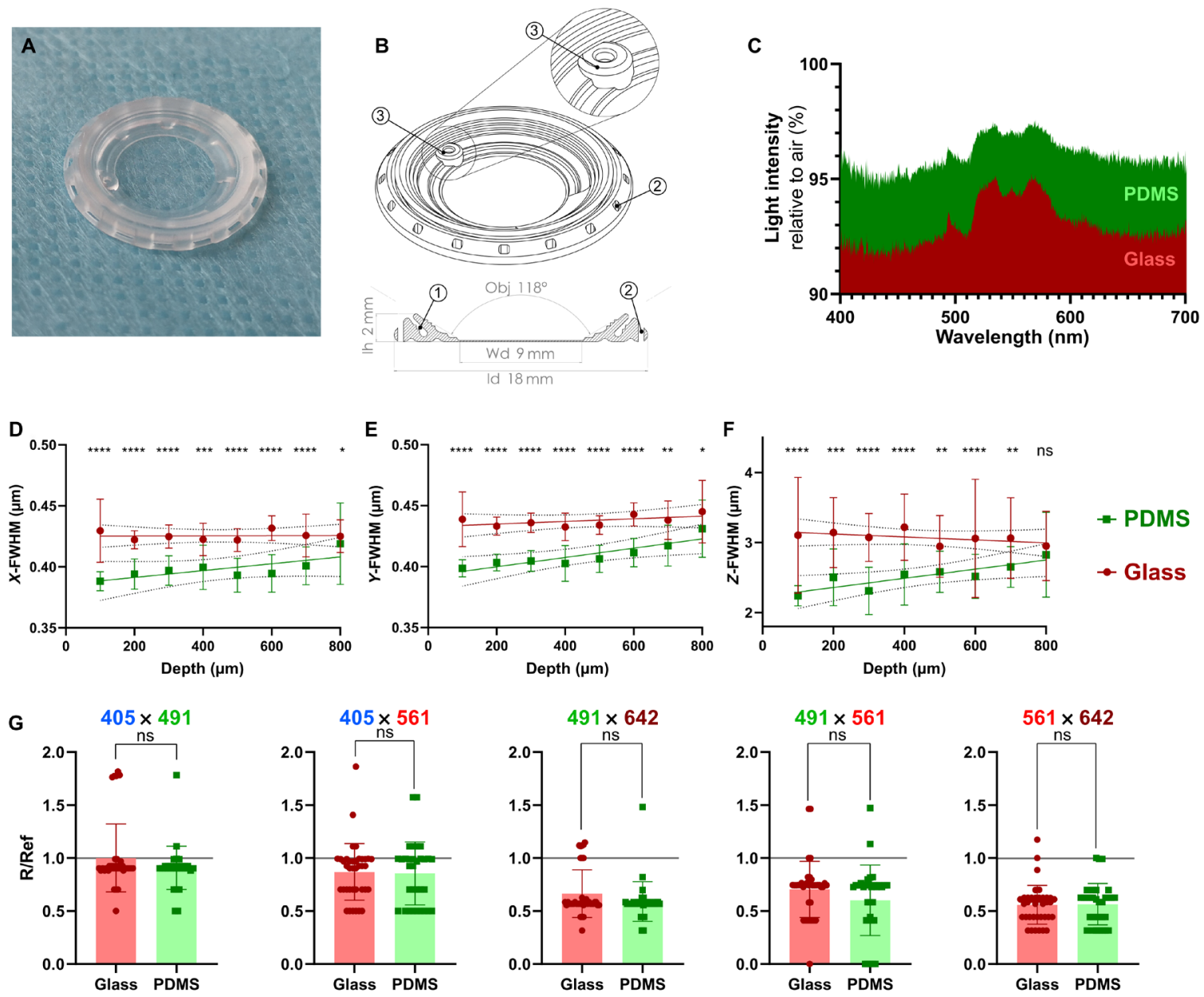

H

Glass

PDMS relaxed

\section{PDMS stretched}
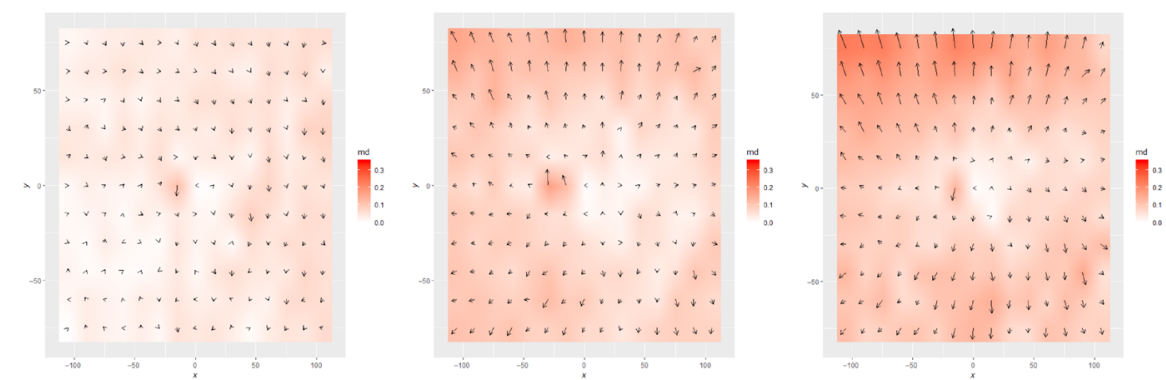

Fig. 1. PDMS implantable devices display microscopy-ready optical characteristics. (A) Photograph of a PDMS intravital imaging window. (B) 3D drawing and cross-sectional view of the window; $18 \mathrm{~mm}$ in diameter (Id) and $2 \mathrm{~mm}$ in height (Ih). The device allows imaging with an angled objective (Obj) up to $118^{\circ}$ over a working diameter (Wd) of $9 \mathrm{~mm}$. At $200 \mathrm{mg}$, the PDMS window is at least $3 \times$ lighter than conventional titanium/glass windows. The angle of the skin groove (1) protects the window frame from damage and removal by the mouse. To promote healing and immobilization of the window, equally spaced holes surround the structure (2). An injection port, $0.7 \mathrm{~mm}$ in diameter (3), is positioned on the side of the window. (C) Light intensity measured through glass (red) or PDMS (green) windows relative to air. (D to F) PSF analysis comparing glass (red) and PDMS (green). Graphs display calculated full width at half maximum (FWHM) values in (D) $X$ axis, (E) $Y$ axis, or (F) $Z$ axis over depth. Comparison between glass and PDMS FWHM at each depth was performed using a Mann-Whitney test ( $n>20$ measurements per section). (G) Chromatic aberration analysis of the indicated color pairs through glass (red) or PDMS (green) windows. Both glass and PDMS in every color pairs are significantly lower than ratio R/Ref $<1$, indicative of colocalization $(P<0.01$, one-sample Wilcoxon text). No significant differences were observed between glass and PDMS for all color pairs $(P>0.05$, Mann-Whitney test). (H) Mapping of the microgrid deformations arising from glass coverslips, relaxed, or stretched (to 200\%) PDMS windows compared to the microgrid alone. Vectors of deformation were calculated on each dot of the grid to generate 2D maps [md, measured deformation norms ( $\mu \mathrm{m})$; vectors represented at $50 \times$ ]. Deformations induced by glass coverslips $(0.037 \pm 0.019 \mu \mathrm{m} \mathrm{SD})$, relaxed $(0.076 \pm 0.031 \mu \mathrm{m} \mathrm{SD})$, and stretched $(0.099 \pm 0.043 \mu \mathrm{m} \mathrm{SD})$ PDMS windows were all below the lateral imaging resolution $\left(0.161 \mu \mathrm{m}\right.$ per pixel) and the inherent aberrations of the optical system. ns, nonsignificant; ${ }^{*} P<0.05 ;{ }^{*} P<0.01 ;{ }^{* * *} P<0.001$; and ${ }^{* * * *} P<0.0001$. All source data are provided in data files S2 and S3. 
adaptable window is centered on a rapid and stereotyped suture-free implantation. To achieve this, we designed a groove around the window circumference that is precisely angled to secure the skin in place in the absence of sutures or glue (Fig. 1B [1]). Passive sealing at the silicone-skin interface maintains the window after implantation, while a series of holes around the frame promotes tissue self-healing for long-term maintenance (Fig. 1B [2]). In addition to securing the skin inside the groove, this design maintains the window in a planar and near-seamless position in line with the animal's body. Windows are therefore poorly accessible to the mouse and surrounding cage enrichment, reducing the risk of dislodgement and damage. The window also includes a dedicated injection port, allowing the local administration of fluids, dyes, or drugs with ease (Fig. 1B [3]).

\section{Optical properties of PDMS-based intravital imaging windows}

The PDMS window is highly transparent, having a refractive index (RI) of $1.38 \pm 0.05-$ in between water $(\sim 1.33)$ and glass $(\sim 1.52)-$ with equivalent light transmittance to glass coverslips of a similar thickness (Fig. 1C and fig. S1, A and B). For decreased light scattering, and thus optimal light penetrance, most IVM studies rely on multiphoton microscopes equipped with pulsed infrared two-photon lasers [two-photon excitation fluorescence microscopy (2PEF)] and water-based objectives for deep tissue imaging $(3,22,23)$. To compare the resolution of the confocal signal through PDMS with conventional glass coverslips, we performed point spread function (PSF) analyses on $0.2-\mu \mathrm{m}$ fluorescent beads embedded in agarose (fig. S1C). The calculated full width at half maximum (FWHM) of signal intensities (fig. S1D) obtained via PDMS substantially outperformed the corresponding glass measurements in lateral ( $x$ and $y$ axes; Fig. 1, D and E) and, particularly, axial planes up to $700 \mu \mathrm{m}$ ( $z$ axis; Fig. $1 \mathrm{~F}$ ), imaging depths routinely achieved by IVM. $X / Y$ ratios of the calculated FWHM for PDMS and glass were also comparable, showing similar means and dispersion close to a ratio of 1 , indicative of perfect $X Y$ squareness (fig. S1E). This implies that potential subtle deformations of the flexible window do not result in microscale geometrical aberrations during imaging. Moreover, using four-color fluorescent beads embedded in agarose (fig. S1F), we did not observe any differences in the degree of chromatic aberrations induced by glass or PDMS, when both materials displayed colocalization $(R /$ Ref $<1)$ of specific channel pairs (Fig. $1 G)$. Finally, as macroscale morphological aberrations arising from the imaging surface are a concern for soft materials, we used a fluorescent stepped microgrid to assess the deformation generated by PDMS windows in a relaxed or stretched state (fig. S1G). While morphological aberrations induced by PDMS windows were marginally more pronounced than that induced by glass coverslips, these were negligible compared with the lateral imaging resolution and the inherent anomalies of the optical system (Fig. $1 \mathrm{H}$ and fig. $\mathrm{S} 1 \mathrm{H}$ ). Collectively, these results validate the PDMS membrane as an appropriate optical material for IVM, which is equivalent, and in some cases superior, to glass for water-based immersion imaging.

\section{Intravital imaging of dynamic, rapidly growing tissues through PDMS imaging windows}

Next, we sought to test the long-term maintenance and imaging performance of our PDMS device in biological contexts where rapid tissue growth or anatomical constraints pose significant challenges for IVM using rigid windows: the branching mammary gland during puberty and pregnancy, patient-derived xenograft (PDX) tumors, and joint muscles. Our optimized surgical protocol (schematically represented in Fig. 2A and described in detail in Materials and Methods) facilitates rapid ( $\sim 5 \mathrm{~min})$ window implantation over superficially located tissues that do not require additional steps for organ exposure. As the device can be folded, the initial incision can be smaller than the circumference of the window for insertion (fig. S2, A and B) and subsequently extended to precisely fit the frame (Fig. 2A). Moreover, prepositioning the window typically takes less than 3 min from the initial incision (fig. S2, A and B), reducing the risk of infection and tissue desiccation before the final window-fitting step (Fig. 2A and fig. S2C). As a result, animals rapidly recover from the short surgical procedure and comfortably maintain windows for up to 35 days (maximum time tested) (fig. S2D and movies S1 and S2). The suture-free implantation procedure does not require advanced surgical skills and can be rapidly conducted by nonexpert users, a significant improvement over conventional windows. As the design is aimed at limiting constrains on the underlying tissues, the flexible window cannot be fixed for imaging. Thus, to stabilize the device and reduce motion artefacts during IVM, we also developed a custom-made holding system compatible with upright microscope configurations and high-numerical aperture ceramic objectives with shallow nose cones (fig. S2, E to H; movie S3; and data file S1).

To assess the compatibility of our device with longitudinal $2 \mathrm{PEF}$ IVM spanning several weeks, we implanted the window over the fourth abdominal mammary gland of pubertal and pregnant $\mathrm{R} 26^{\mathrm{mTmG} /+}$ reporter mice (24), where all cells are labeled with membrane tdTomato fluorescence (Fig. 2, B to D, and fig. S3, A to D). Despite substantial increases in body size during puberty, PDMS windows were well maintained over time, allowing specific tissue regions to be recognized in successive imaging sessions (Fig. 2, C and D). Window implantation at late pregnancy also allowed the flexibility of our device to be tested under extreme conditions of skin stretching and rapid body growth (corresponding to a $10 \%$ increase over 3 days) (fig. S3A). In this context, we could visualize lobuloalveolar development in the prelactating mammary gland at high cellular resolution (fig. S3, B and C). Moreover, implanted windows had no detrimental impact on normal parturition or the lactational competence of the underlying mammary gland (fig. S3D). Occasionally, IVM after implantation revealed an accumulation of immune cells underneath the window that obscured underlying epithelial structures (fig. S3E). This was also reported with conventional glass/titanium imaging windows $(6,13)$, suggesting that local inflammatory reactions may occur in response to surgery. Under our standardized surgical procedure and pre/postoperative care regimens (see Materials and Methods), immune cell accumulation typically persisted for approximately 2 to 3 days after surgery. To mitigate this issue, we performed saline washes before imaging via the integrated window injection port (Fig. 1B [3]), which provides a low-risk and noninvasive method for conserving image quality over time (fig. S3E and movie S4).

Next, we evaluated the performance of our device over rapidly growing PDXs from Ewing sarcomas engrafted in the interscapular region of immunodeficient NMRI-Nude and $\mathrm{Rag}^{\mathrm{KO} / K O-G F P}$ mice $(25,26)$. PDMS windows implanted over tumors in this location were well tolerated by both strains (Fig. 2E, fig. S3F, and movie S5). To enable high-resolution fluorescence IVM of tumors, we generated 


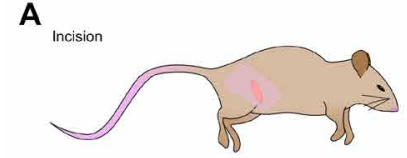

Window folding
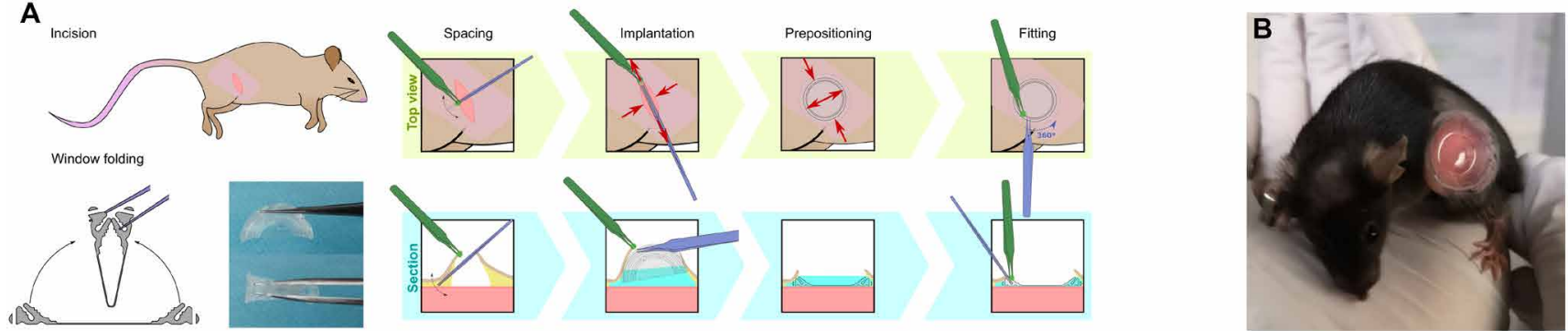

C
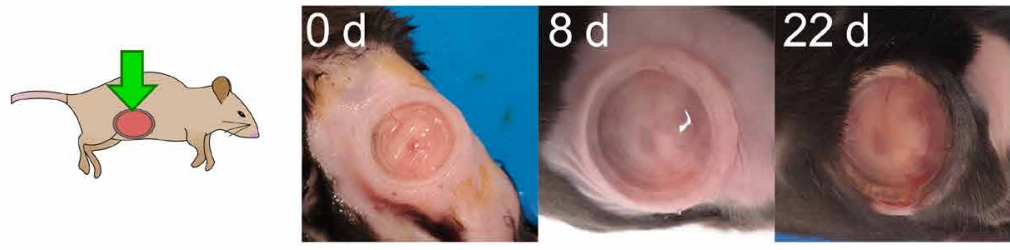

E
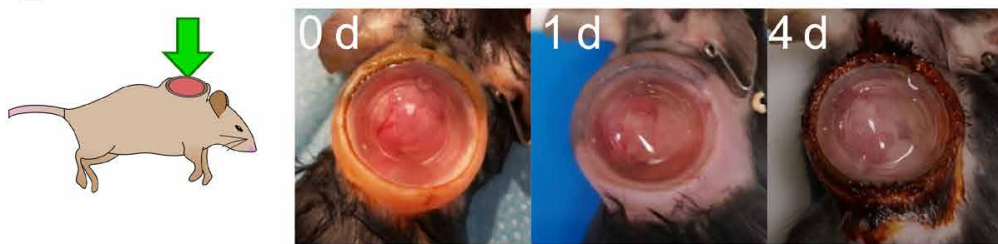

G

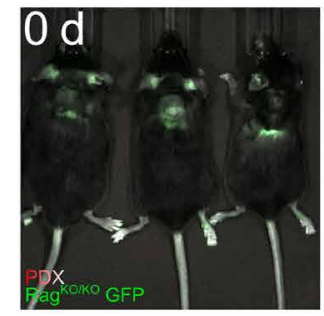

H

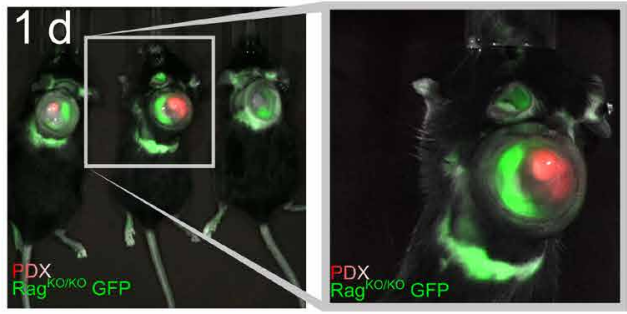

D

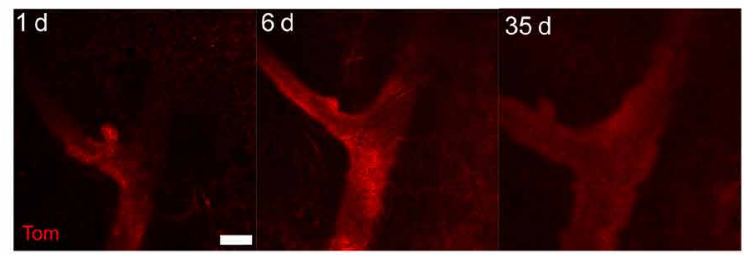

F

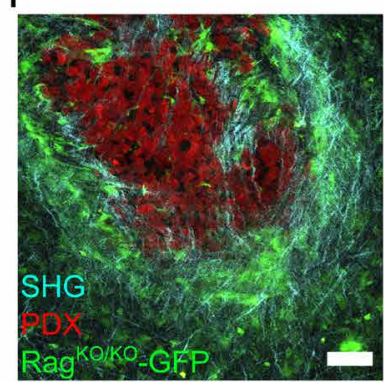

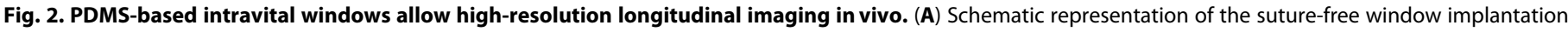

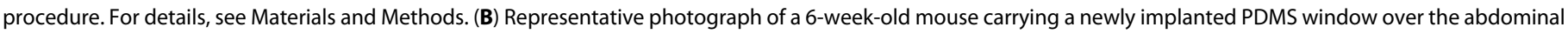

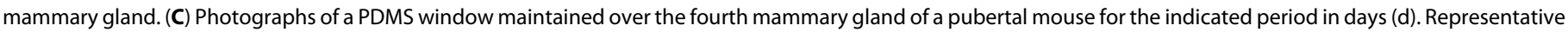

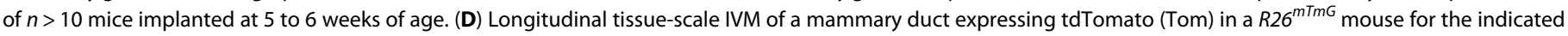

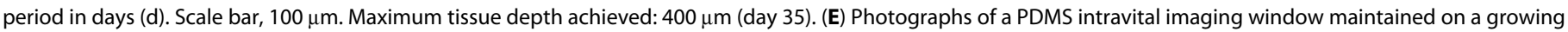

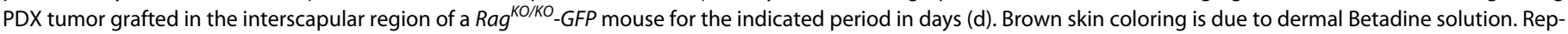

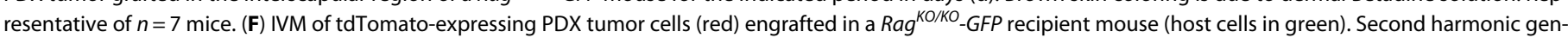

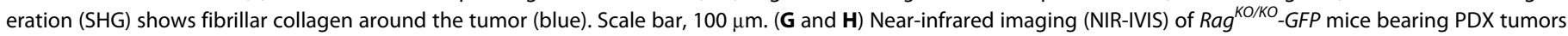
expressing tdTomato fluorescence before $(\mathrm{G})$ or after $(\mathrm{H})$ window implantation.

tdTomato-expressing PDX tumor cells and grafted them in $\mathrm{Rag}^{\mathrm{KO} / K O-G F P}$ host mice. Windows were subsequently implanted over tumors approximately $400 \mathrm{~mm}^{3}$ in size, which continued to grow rapidly underneath the device at a daily rate of 100 to $200 \mathrm{~mm}^{3}$ until reaching their defined endpoint size (Fig. 2E). Despite the anatomical constraints posed by this implantation site, we were able to obtain stable and high-quality IVM images of tumor cells (red), surrounding host stromal cells (green), and the encapsulating tumor collagen network [second harmonic generation (SHG), blue] through PDMS windows (Fig. 2F). PDXs are commonly used in preclinical studies, which typically rely on near-infrared (NIR) imaging systems [e.g., In Vivo Imaging System (IVIS)] for monitoring tumor responses over time. However, these systems are poorly compatible with fluorescence imaging, and, as expected, standard IVIS imaging failed to detect tdTomato fluorescence in our PDXs models (Fig. 2G). In contrast, a clear and well-defined signal was detected for both green (host cells) and red (tdTomato-expressing tumor cells) fluorescence after PDMS window implantation (Fig $2 \mathrm{H}$ ). Thus, the application of our PDMS window in this context enables increased reporter sensitivity in addition to the prescreening and 
prioritization of mice for downstream high-resolution confocal microscopy, representing a valuable improvement to canonical NIR studies.

\section{IVM of muscle regeneration through PDMS imaging windows}

Finally, to test other body locations that require high levels of flexibility, we implanted the window over the mouse's lower back and thigh muscles (Fig. 3, A and B). These anatomical positions are uneven and adjacent to joints, leading to continual deformation of the window and its frame in multiple axes with body movement. While typically problematic with conventional rigid imaging devicesrestricting studies to acute or short-term imaging (27-29) - our implanted PDMS windows were well maintained at these locations for at least 3 weeks (maximum time examined). To validate the utility of the device for visualizing dynamic biological processes over time, we sought to monitor stem cell activation during injury-induced muscle regeneration in $\mathrm{Pax}_{7 \mathrm{CreERT2/+}} ; R_{2} 6^{m T m G /+}$ mice $(24,30)$. Tamoxifen administration in this model induces permanent green fluorescent protein (GFP) labeling of Pax7-expressing muscle stem cells and their progeny, allowing their dynamic behaviors in response to muscle injury to be visualized in situ. To induce muscle injury in $P a x 7^{\mathrm{CreERT2/+}} ; \mathrm{R}^{\mathrm{m}} \mathrm{m}^{\mathrm{mTG} /+}$ mice, cardiotoxin was administered by intramuscular injection either during the window implantation procedure or after, using the inbuild window injection port to test the feasibility of accurate tissue targeting underneath the device. High-resolution IVM imaging through the PDMS window revealed the movement and division of $\mathrm{GFP}^{+}$cells at 3 days after injury (Fig. 3C and movie S6), a time point at which muscle stem cells are maximally proliferating $(29,31)$. High-resolution images could be acquired through PDMS windows with no observable decline in image quality for at least 18 days after injury (Fig. 3D), allowing the process of muscle regeneration to be visualized in its entirety. Thus, our device is ideally suited to high-resolution longitudinal IVM at the cell and tissue scale of dynamic processes that occur over prolonged time frames, including in challenging anatomical sites where rigid windows cannot be maintained long term.

\section{DISCUSSION}

Dynamic visualization of cellular processes unfolding inside living animals is essential for an in-depth understanding of normal and pathological organ function. A major challenge thus far has been the ability to study cellular dynamics in vivo without perturbing tissue physiology. Intravital imaging represents a critical advance in this respect. Nevertheless, the ability to concomitantly expose and preserve tissues for long-term imaging remains a fundamental challenge, one largely inaccessible to most laboratories. The development of a flexible, transparent, and seamlessly integrated "second skin" - as afforded by our imaging window-represents a marked advance in methodology. Our sutureless PDMS-based device has significant technical, biological, and ethical advantages over conventional imaging windows. This includes a portal for ad hoc local delivery of substances in addition to a rapid and straightforward implantation. The lightweight and low-profile design of the window also ensures minimal impact on normal animal behaviors in addition to facilitating group housing and the safe inclusion of environmental enrichment, an important improvement on current welfare protocols. Moreover, implantation of PDMS imaging windows leads to substantial improvements in the performance of NIR imaging systems for in vivo fluorescence detection. This window is also compatible with other imaging technologies, including magnetic resonance imaging (MRI) and echography. Thus, our device is
A
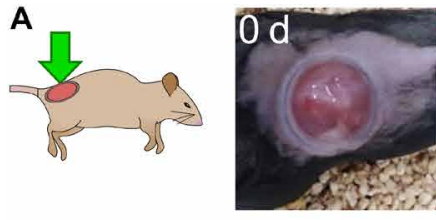

$18 d$
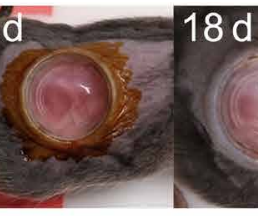

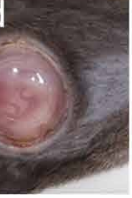

C
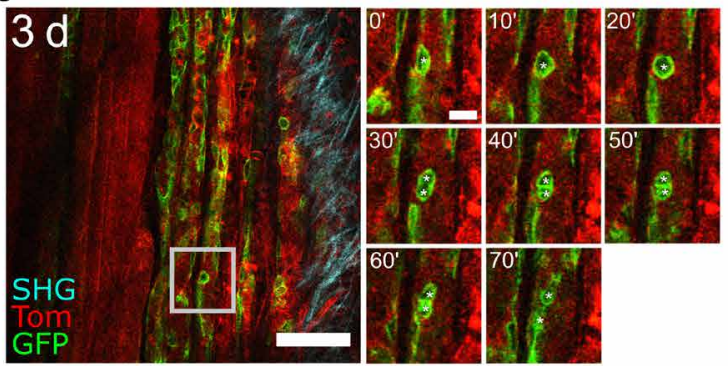
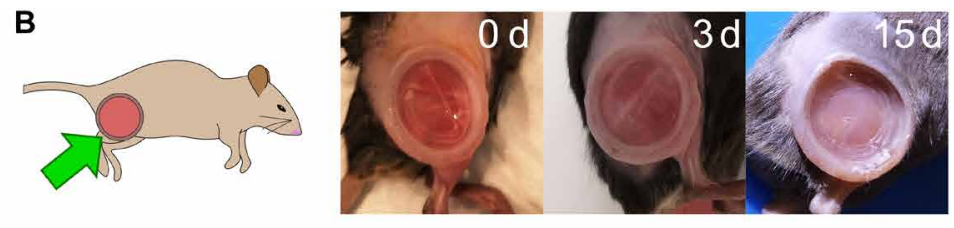

D
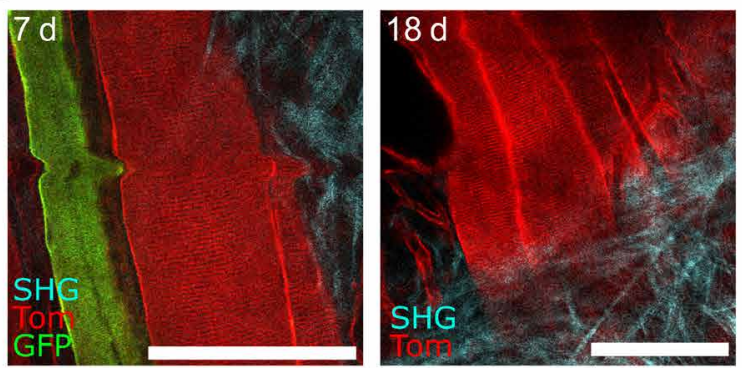

Fig. 3. PDMS-based intravital windows allow high-resolution longitudinal imaging of injury induced muscle regeneration in vivo. (A and B) Representative photographs of a PDMS intravital imaging window maintained on the back (A) or thigh (B) muscle of a mouse for the indicated period in days (d). Brown skin coloring at day 1 in (A) is due to dermal Betadine solution. Representative of $n=9$ mice implanted on the back or thigh muscles. (C) IVM of thigh muscle regeneration through a PDMS imaging window 3 days ( $3 \mathrm{~d}$ ) after cardiotoxin-induced muscle injury in $P a x 7^{\text {CreERT2}} ; R 26^{m T m G}$ adult mice. All cells were labeled with membrane tdTomato fluorescent protein (Tom, red). Tamoxifen-induced Cre-mediated enhanced GFP protein expression (green) in Pax7-positive muscle stem cells and their progeny. SHG imaging shows fibrillar collagen organization in blue. Time-lapse imaging shows muscle stem cell division and migration (white asterisks) over $70 \mathrm{~min}$. Scale bars, $100 \mu \mathrm{m}$. (D) Representative IVM images of muscle fibers 7 and 18 days after implantation in a $P a x 7^{\text {CreERT2 }} ; R 26^{m T m G}$ mouse, showing resolution at the cellular scale and maintenance of excellent imaging quality over time. Scale bars, $100 \mu \mathrm{m}$. The small bump in the image at 7 days is due to the breathing-induced movement during image acquisition. 
ideally suited for developmental, pathological, or pharmacokinetic studies across multiple imaging scales.

While particularly suited to superficial tissues in dynamic and flexible locations, the versatility of PDMS makes our model readily adaptable for internal organ imaging by incorporating additional surgical steps for organ exposure or silicone-based glues. The suppleness of the device, however, renders it less suitable for implantation over rigid tissue sites, such as for brain or thoracic organ imaging that demands more specialist solutions $(10,18,19)$. Nonetheless, silicone mass or surface modifications, in addition to drug embedding, can be used to alter the window's mechanical, adhesive, or bioactivity properties for enhanced healing, tissue anchoring, permeability, and resistance to biological/chemical agents. As substrate rigidity plays important roles in tissue development and tumorigenesis (32), the more physiological stiffness of PDMSalongside the ability to tune its mechanical properties for specific research needs - is also an important advantage over conventional glass coverslip models. Moreover, in light of exponential progress in microfluidic devices using the same production methods, integrating micropatterns, electrodes, or surface modifications into the window design for live sensing or interaction with the underlying tissue represents a realistic future advance. Finally, in contrast to currently available solutions, plastic engineered devices such as ours are scalable, allowing single-use and widespread distribution at low cost for basic and preclinical research.

\section{MATERIALS AND METHODS Animals}

All studies and procedures involving animals were in strict accordance with the recommendations of the European Community Directive (2010/63/UE) for the protection of vertebrate animals used for experimental and other scientific purposes. The project was specifically approved by the ethics committees of Institut Curie CEEA-IC \#118 and Institut Pasteur (reference \#2015-0008) and by the French Ministry of Research (authorization references \#0424003, \#133102018020112578233-v1, \#6354-2016080912028839-v4, \#112062017090816044613-v2). We comply with internationally established principles of replacement, reduction, and refinement in accordance with the Guide for the Care and Use of Laboratory Animals (National Research Council 2011). Husbandry, supply of animals, as well as mouse maintenance and care in the Animal Facility of Institut Curie (facility license \#C75-05-18) before and during experiments fully satisfied animal needs and welfare. Suffering of the animals was kept to a minimum.

The following mouse lines were used in this work: $R 26^{m T m G}$ mice (24), NMRI-Foxn $1^{n u / n u}$ (purchased from Janvier Labs), Pax $7^{\text {CreERT2 }}$ mice (30), and $\mathrm{Rag}^{\mathrm{KO} / \mathrm{KO}}$-GFP mice [B6(Cg)-Rag2 $\left.{ }^{\text {tm1.1Cgn }} / \mathrm{J} ; \mathrm{Tg}(\mathrm{UBC}-\mathrm{GFP})(25,26)\right]$. All experiments were performed in 4- to 8-week-old female mice.

\section{Prototyping}

Three-dimensional (3D) conception of the window, mold, and holder was performed using SOLIDWORKS 2018 (Dassault Systèmes). 3D printing of window holder prototypes was performed using a homemade system [Fused deposition modeling (FDM)] and MultiJetFusion [Hewlett-Packard (HP)] using various materials, predominantly generic acrylonitrile butadiene styrene (FDM) and polyamide and polypropylene (PP) (fusion). We recommend PP fusion for higher printing resolution, temperature, and hygroscopic stability.

\section{Window production}

Biocompatible transparent silicone (Elkem) components (A and B) were mixed at a $1: 1$ ratio $(A: B)$ according to manufacturer guidelines for $5 \mathrm{~min}$ per $50 \mathrm{~g}$ of mix. The mix was then degassed for 5 and $10 \mathrm{~min}(<10 \mathrm{mbar})$ with a vacuum release in between to maximize air removal. Air removal was completed by centrifuging the mix at $1000 \mathrm{~g}$ for $5 \mathrm{~min}$. The degassed silicone mix was kept for a maximum of 24 hours at $4^{\circ} \mathrm{C}$ to prevent spontaneous polymerization. Windows were produced using a custom three-stage single footprint compression mold made of machined steel, which was maintained at $100^{\circ} \mathrm{C}$ throughout molding. Approximately $1 \mathrm{ml}$ of silicone was placed on the footprint before closing the mold and applying compression at 5 tons for $10 \mathrm{~min}$. Polymerized windows were then postcured for at least 24 hours at $60^{\circ} \mathrm{C}$ and subsequently washed in absolute ethanol before autoclaving. While untested to date, implant sizes may be modified (e.g., smaller versions for specific tissue sites) subject to optimizing elastomer stiffness to preserve the mechanical properties of the device required for suture-free maintenance.

\section{Optical characterization of PDMS windows}

Optical, chromatic, and morphological aberrations depend on the optical path, which includes the objective and microscope core. Therefore, all assays were performed using a single microscope setup and water as the imaging medium. All measurements were acquired using a $40 \times / 1$ numerical aperture (NA) Water DIC PL APO VIS-IR objective on an upright spinning disk microscope (CSU-X1 scan-head from Yokogawa; Carl Zeiss, Roper Scientific, France), equipped with a CoolSNAP HQ2 charge-coupled device (CCD) camera (Photometrics) and MetaMorph software.

\section{Optical aberrations (PSF)}

To measure the PSF representing optical aberrations arising from RI changes, we prepared 2\% agarose gels containing 1:200 fluorescein and 1:400 diluted FluoSpheres carboxylate-modified microspheres $(0.2-\mu \mathrm{m}$ diameter $)$. For both glass and PDMS windows, we acquired eight stacks of $100 \mu \mathrm{m}$ with a step size of $0.2 \mu \mathrm{m}$ at increasing depths from the window/gel interface ( $\mathrm{Z} 0$, defined using the background signal in the fluorescein channel). PSF was calculated using the MetroloJ plugin (33) in Fiji (ImageJ v1.53) on at least 20 beads per section.

\section{Chromatic aberrations}

To measure the chromatic aberrations representing optical aberrations induced by wavelength-specific RI changes, we prepared $2 \%$ agarose gels containing TetraSpeck Microspheres (4- $\mu \mathrm{m}$ diameter) diluted at 1:400. Data were acquired over $200 \mu \mathrm{m}$ from the first bead detected in $\mathrm{Z}$ for both glass and PDMS windows. Laser intensities and exposures were set to obtain similar intensity range (12 bits). $\mathrm{R}$ and Rref were calculated using the MetroloJ plugin (33) in Fiji (ImageJ v1.53) on at least 30 beads per condition.

\section{Morphological aberrations}

To measure morphological aberrations that may arise from an uneven window surface, we imaged a microgrid (Argolight SLG-075, pattern B) using a laser excitation wavelength of $405 \mathrm{~nm}$ through either water only (uncovered), glass, relaxed PDMS, or stretched (to 200\%) PDMS (wavy) window in duplicates. Dot grids were segmented using Fiji v1.53 (threshold > analyze particles), and dot coordinates were extracted. Using a central motif (cross $0 ; 0$ ) and the top left dot $(-105 ;-75)$, we corrected the rotation of the grid under the different materials and compared the corrected coordinates 
of each dot to either "medium only" (measured) or the theoretical $(15 \times 15 \mu \mathrm{m})$ pattern B matrix. Graphical deformation maps were generated using the R package ggplot2 (34).

\section{Window implantation}

Before surgery, all instruments were sterilized by autoclaving or heat sterilization. PDMS windows were sterilized by autoclaving to avoid darkening. To ensure sterility throughout the procedure, mouse preparation and handling, adjustment of anesthesia flow rates, and similar tasks were performed by an assistant, limiting the surgeon to only contacting sterile surgical tools and the surgical field. The physiological body temperature of the anesthetized mouse was maintained throughout the procedure using a heated induction chamber and heated surgical station.

\section{Preparation (performed by the surgical assistant)}

Preoperative care. Before anesthesia, mice were administered $125 \mathrm{mg} / \mathrm{kg}$ of Noroclav $(140 \mathrm{mg} / \mathrm{ml}$ of amoxicillin; $35 \mathrm{mg} / \mathrm{ml}$ of clavulanic acid) and an analgesic cocktail $[0.1 \mathrm{mg} / \mathrm{kg}$ of buprenorphine (Buprecare) and $5 \mathrm{mg} / \mathrm{kg}$ of carprofen (RIMADYL)] by subcutaneous injection. Analgesics were administered into the neck skin fold for all experiments apart from implantation in PDX models, where they were administered in the rear leg skin fold.

Surgical field preparation. Mice were anesthetized in an induction chamber using $4 \%$ isoflurane (ISOFLURIN). Once anesthetized, mice were transferred onto a heat pad, and anesthesia was maintained using an isoflurane concentration of 1.5 to $2 \%$. Ophthalmic ointment was applied to eyes to prevent corneal drying. At the planned implantation site, mouse hair was trimmed using an electronic pet clipper (Aesculap Exacta). Residual hairs were removed using depilatory cream for a maximum of 2 min to minimize the risk of skin damage and drying. Stray hairs and cream were removed using Betadine Scrub $4 \%$ before a soap rinse with sterile water. The shaved skin and surrounding areas were subsequently disinfected using dermal Betadine 10\%. Finally, the prepared surgical area was covered by precut sterile film (Tegaderm).

\section{Implantation (performed by the surgeon)}

We recommend the following surgical tools to limit skin and tissue damage: Graefe extra fine straight $1 \times 2$ teeth forceps [for skin and membranes; Fine Science Tools (FST) \#11153-10], flat blunt forceps (for the window and tissue), serrated blunt forceps (for the window), fine and sharp straight scissors (for skin and membranes; FST \#14568-09), and Strabismus blunt straight scissors (for blunt dissecting the subcutaneous pocket; FST \#14574-09).

To implant the PDSM window $(\varnothing 18 \mathrm{~mm})$ in the prepared surgical area, a $10-\mathrm{mm}$ incision was made in the skin overlying the tissue of interest [corresponding to approximately $15 \mathrm{~mm}$ when stretched (Fig. 2A)]. Next, using blunt scissors, the skin was carefully detached from underlying tissues approximately 5 to $10 \mathrm{~mm}$ around the incision to generate sufficient space for the implanted window. Throughout the procedure, prewarmed saline solution (sterile $0.9 \%$ $\mathrm{NaCl}$ ) was used to prevent tissue dehydration. Before positioning the window, the incision site was filled with prewarmed saline solution to minimize the risk of introducing air underneath the window during implantation. Using thin forceps with blunt and flat edges, the PDMS window was folded in half and placed into the incision pocket where it was unfolded under the skin. Typically, the procedure from the first incision to prepositioning of the window under the skin was performed in less than 3 min (fig. S2, A and B). To fit the window in place, the skin edges were positioned into the window groove using two forceps that alternate between holding the upper frame and sliding the skin around the window (similar to the technique for adjusting a tire on a rim). During this step, the incision size can be adjusted, if required, to tightly seal the skin around the window. Experienced users are capable of performing the entire surgical procedure in $\sim 5 \mathrm{~min}$.

Postoperative care. After surgery, mice were placed in a prewarmed cage (on a heating pad) to recover from the anesthesia. Mice were closely monitored for signs of pain, discomfort, and infection after window implantation. The second and last Noroclav antibiotic dose was provided by subcutaneous injection the day after surgery. The skin surrounding the window was cleaned using dermic Betadine $1 \%$ every 2 to 4 days to prevent infection. Ibuprofen was administered in drinking water $(0.4 \mathrm{mg} / \mathrm{ml})$ for 7 days after surgery to minimize inflammation and pain. If signs of pain are observed, then postoperative analgesics $[0.1 \mathrm{mg} / \mathrm{kg}$ buprenorphine (Buprecare) and $5 \mathrm{mg} / \mathrm{kg}$ of carprofen (RIMADYL)] can be administered by subcutaneous injection the day after surgery.

Mice bearing windows were group housed where possible after surgery and provided with cage enrichment suitable for postsurgery recovery, including nesting material, housing, and chew sticks. Recovery food (DietGel Recovery, ClearH20) was provided in the cage to limit mechanical stress from reaching the feeder. Singly housed mice without sufficient environmental enrichment may be more likely to damage the device during the first 1 to 2 days after surgery. Stringent aseptic surgical technique, and including preventative antibiotic and ibuprofen treatment, minimizes this risk by reducing inflammation and irritation. Overall, younger animals were more prone to skin damage arising from the procedure, which may be due to the window/animal size ratio and/or increased activity at earlier ages. Cage cleanliness did not appear to affect the risk of implant infection. Typically, mice exhibited minor weight loss the day after surgery $(-4.20 \pm 4.74 \%$, within ethical limits), which were restored by day $2(+2.93 \pm 3.18 \%)$. As expected, PDMS windows retain their dimensional characteristics after implantation, displaying no discernible swelling as a result of marginal exposures to biological fluids during study time frames (maximum 35 days tested).

Handling window-bearing mice. When the implantation is performed correctly with stringent asepsis, no mouse should spontaneously lose their implanted windows. Windows are at a risk of displacement, however, in the first few days after surgery when handling mice using conventional restraining techniques. Thus, we recommend adapting handling techniques when assessing mice in the days immediately after window implantation and consider the use of light gas anesthesia where possible, e.g., when performing procedures such as intraperitoneal injection.

\section{Troubleshooting guide for the implantation procedure (A) Air is trapped under the window during implantation}

Prepare a $1-\mathrm{ml}$ syringe with sterile, prewarmed $0.9 \% \mathrm{NaCl}$, and a 30 -gauge needle. Position the mouse so that the injection port is facing upward and disinfect it with $70 \%$ ethanol. Using fine forceps, hold the injection site and inject 200 to $500 \mu \mathrm{l}$ of saline solution underneath the window: The air bubble(s) will move close to the injection site. Slowly aspirate using the syringe to remove air and as much of the saline solution as possible (as demonstrated in movie S4). Dry the injection port. Wait for the saline solution to be absorbed prior to imaging. 


\section{(B) Air appears under the window after implantation}

During surgery, take care to make a straight incision. Irregular and ragged incisions increase the risk of air leakage. Check the window integrity: If the window is perforated, then it must be discarded. Check carefully the skin in the groove for breakages as small cuts can cause air leakiness. If a small break or nick ( 1 to $2 \mathrm{~mm}$ maximum) is observed, then place a small amount of glue on the groove to seal the leakage and proceed to (A). The next day, check carefully for the absence of air bubbles.

\section{(C) Presence of small amount of fluid (exudate) and immune} cells between the tissue and the window

This is common for a few days after surgery and should disappear after 2 to 3 days. To minimize this risk, review the surgery procedure to ensure stringent asepsis, check/change the ibuprofen in the drinking water, and ensure that food is accessible to limit mechanical stress. If the exudate precludes imaging, then administer prewarmed saline through the injection port to flush underneath the window [see (A); fig. S3E]. As immune cells may return to the site after flushing in the days following surgery, this step may need to be repeated in a subsequent acquisition session, depending on the desired imaging frequency.

\section{(D) Hair underneath the window}

Review the preparation step. Trim a larger area if necessary. A spray plaster can be used before incision to trap residual stray hairs.

\section{(E) Skin becomes thinner or dry around the window}

If this issue occurs within the first 7 days from implantation, then reduce depilatory cream incubation and/or change the brand. Use $1 \times 2$ teeth forceps to reduce skin damage. Limit damage to vasculature when performing blunt dissection to detach the skin. This issue may appear after long-term maintenance ( $>2$ weeks), but, in our experience, it did not affect imaging (no infection, no air bubbles) when postsurgery care was properly performed.

\section{(F) Skin or tissue become necrotic}

Immediately terminate the experiment. This should never happen; review the surgery procedure (including asepsis, tissue drying, and damage to vasculature).

\section{Intravital imaging through PDMS windows}

Imaging on the day of implantation is not recommended to avoid additional stress to the surgery area and overconstraining the window. Residual physiological saline under the window immediately after implantation can also affect imaging depth. Instead, perform imaging the next day (day 1) at the earliest. While the mouse is anesthetized, administer postoperative antibiotics by subcutaneous injection. Carefully inspect the device and clean the surgical area as described above. Mice are gas anesthetized using $4 \%$ isoflurane (ISOFLURIN) initially and maintained using 1.5 to $2.5 \%$ isoflurane for short-term imaging. When performing time-lapse imaging spanning several hours, reduce the isoflurane concentration to between 0.8 and $1.2 \%$. These anesthesia levels are optimal for long-term maintenance of mice in a nonresponsive state with a slow, constant, and nonforced breathing pattern. Irregular and abnormal breathing patterns are associated with persistent anesthesia greater than $1.5 \%$, which perturbs imaging (increases motion-induced image deformations) and can decrease survival times (35). Fast breathing and reflex responses indicate insufficient anesthesia, which also disturbs imaging and increases the risk of the mouse awakening during imaging.

Before imaging. To maintain animal hydration for short-term imaging ( $<2$ to 3 hours), administer $250 \mu \mathrm{l} / 10 \mathrm{~g}$ of saline solution by subcutaneous injection after anesthesia induction. In experiments exceeding 3 hours, mouse hydration can be maintained during imaging using a subcutaneous or intraperitoneal infusion of glucose and electrolytes ( $\sim 50$ to $100 \mu \mathrm{l} /$ hour $)$ via an indwelling line. Our custom-made holder, adapted for upright microscopes (ready to print STL files provided in data file S1), permits regional body immobilization through compression. A rigid conical structure (containing immersion medium, usually water) presses on the edge of the window, allowing the tissue and window to be exposed and stretched beneath the objective as the mouse rests on a foam bed, which preserves breathing while cushioning movements. The mouse is positioned on the imaging holder and gently pushed on the static stage, centering the window in the conic aperture. The mouse should be tightly constrained; the foam absorbs excessive compression. The mouse is then observed for $5 \mathrm{~min}$ to check breathing and the stability of the anesthesia. Before placing the holder onto the microscope stage, the conic aperture is filled with 2 to $3 \mathrm{ml}$ of pure water. The holder may be adapted for inverted microscope configurations, using gel-based immersion medium instead of water for imaging. Critical: Do not use oil-based imaging medium with PDMS windows, as hydrophobic solvents may result in window swelling, fragility, and toxicity to underlying tissues. Placing a glass coverslip over the PDMS window may enable the use of oil-based objectives for imaging superficial tissue structures, provided that the underlying material remains protected throughout.

During imaging. Find structures of interest using brightfield or fluorescence with the oculars. During scanning, optimize laser power and exposure times to minimize phototoxicity and pixel saturation. PDMS windows are resistant to high-power laser bursts and are therefore compatible with localized photobleaching and photoablation applications.

After imaging. The imaging medium must be removed from the conic aperture using a paper towel. The mouse is released from the holder and placed on a heated pad (set at $\left.38^{\circ} \mathrm{C}\right)$ for recovery. During this time, the window is cleaned using a paper towel and $70 \%$ ethanol, and the surrounding skin is disinfected with dermic Betadine $1 \%$.

\section{Troubleshooting guide for imaging procedures (G) The window cannot be correctly positioned on the conic aperture}

Use softer foam in the holder that allows fitting to any position.

\section{(H) The imaging medium (water) leaks}

The conic aperture is not centered on the window. Reposition it or use a gel-based imaging medium.

\section{(I) The holder disturbs breathing}

Reduce the isoflurane dose. Reduce the compression on the animal. Sculpt the foam, ensuring that the mouse's airway is not restricted. Chisel the foam to modify the mouse position (a horizontal plane may not be optimal for the tissue of interest).

\section{(J) Poor visibility during imaging}

Exudate accumulation and/or epithelioid "membrane" formation beneath the window (which can develop with long-term maintenance) can interfere with imaging. This has also been observed with glass/titanium windows $(6,13)$ and is likely due to inflammatory reactions in response to surgery. To minimize this risk, review the surgical procedure, taking care to limit tissue damage and maintain stringent asepsis throughout. While the epithelioid membrane-like structure affects tissue imaging depths, overall, it has no detrimental impact on image quality. However, when coupled with immune 
cells and/or fibrosis, it can preclude imaging. To alleviate this issue, prewarmed saline can be administered via the injection port to flush underneath the window [following the protocol in (A); fig. S3E and movie S4). Alternatively, reposition the window on a different area of the tissue of interest or terminate the experiment.

\section{Intravital microscopy}

Animals were anesthetized and positioned for imaging using the custom-made holder as described above. Intravital imaging was performed on an upright Nikon A1R MP multiphoton confocal microscope equipped with a pulsed Spectra-Physics Insight DeepSee laser (680 to $1300 \mathrm{~nm}, 120$-fs pulses with auto-alignment), Luigs \& Neumann $X Y$ motorized stage and four GaAsP nondescanned detectors with SP492, BP 525/50, BP 575/50, and BP 629/56 filter sets. The microscope was surrounded by a heated dark box maintained at $38^{\circ} \mathrm{C}$. All images were acquired using $16 \times \mathrm{NA} 0.8$ or $25 \times \mathrm{NA}$ 1.1 Plan Apo LambdaS water objectives. An excitation wavelength of $960 \mathrm{~nm}$ was used for GFP and TdTomato in addition to SHG imaging of collagen.

\section{Image processing and visualization}

Time-lapse acquisitions were corrected for movements and drifts using the "Correct 3D Drift" plugin (36) in Fiji (ImageJ v1.53) using the SHG channel as a reference.

\section{Generation of tdTomato-expressing PDX tumor cells}

Ewing sarcoma PDX tumors (IC-pPDX-87) were surgically removed and enzymatically dissociated to single cell level using the protocol described in Stewart et al. (37). Cells were resuspended $\left(2.25 \times 10^{7}\right.$ cells per $1.5 \mathrm{ml}$ ) in Dulbecco's modified Eagle's medium/F12 supplemented with 1\% penicillin-streptomycin and 2\% B27 (all from Thermo Fisher Scientific) and transduced overnight with a TdTomatolentivirus in a 2-ml tube using an Intelli-Mixer RM-2L (Dutcher), program $\mathrm{F} 1,5$ revolutions per minute, in a $37^{\circ} \mathrm{C}$ incubator with $5 \%$ $\mathrm{CO}_{2}$. TdTomato-lentivirus was generated by replacing the GFP sequence with tandem Tomato in the Lenti-sgRNA-GFP plasmid (Addgene plasmid no. 65656; http://n2t.net/addgene:65656; a gift from C. Vakoc). The next day, cells were spun at $500 \mathrm{~g}$ for $10 \mathrm{~min}$. For one injection, $1 \times 10^{7}$ cells were resuspended in $50 \mu \mathrm{l}$ of media and kept on ice. Fifty microliters of Matrigel (Corning, ref. 354234) was added to the cell suspension before injection into the interscapular fat tissue using an insulin syringe (BD, ref. 324891).

\section{Muscle regeneration studies}

Muscle injury was performed in $\operatorname{Pax} 7^{\mathrm{CreERT2}} ; R 26^{m T m G}$ adult mice at the time of PDMS imaging window implantation by intramuscular injection of $50 \mu \mathrm{l}$ of cardiotoxin ( $1 \mu \mathrm{M}$; Latoxan, L8102) diluted in $0.9 \% \mathrm{NaCl}$. Cardiotoxin was administered either before window positioning or after through the injection port. Mice received $3 \mathrm{mg}$ of tamoxifen free base (Euromedex) by intraperitoneal injection 2 days before intravital imaging.

\section{IVIS imaging}

NIR fluorescence imaging was performed using a highly sensitive CCD camera mounted in a light-tight specimen box (IVIS 50; Living Image version: 4.3.1.0.15880). Animals were anesthetized in a warm induction chamber using $4 \%$ isoflurane and subsequently placed onto a warmed stage inside the camera box and supplied with 1.5 to $2 \%$ isoflurane to maintain anesthesia during imaging.
The emitted fluorescence signal was detected by the IVIS camera system, integrated, digitized, and displayed. Excitation filters for Tomato and GFP fluorescence were 535 (DsRed filter, position 3) and 465 (GFP filter, position 2), respectively. Exposure times and pixel binning were auto-optimized for each fluorescence channel to minimize overexposure and normalized for comparison using the formula normalized_intensity = (measured_intensity/exposure_time) $/$ binning_factor ${ }^{2}$. Raw parameters are provided in data file S2.

\section{SUPPLEMENTARY MATERIALS}

Supplementary material for this article is available at http://advances.sciencemag.org/cgi/ content/full/7/25/eabg7663/DC1

View/request a protocol for this paper from Bio-protocol.

\section{REFERENCES AND NOTES}

1. M. J. Pittet, R. Weissleder, Intravital imaging. Cell 147, 983-991 (2011).

2. G. Follain, L. Mercier, N. L. Osmani, S. Harlepp, J. G. Goetz, Seeing is believing-multi-scale spatio-temporal imaging towards in vivo cell biology. J. Cell Sci. 130, 23-38 (2017).

3. B. Lloyd-Lewis, Multidimensional imaging of mammary gland development: A window into breast form and function. Front. Cell Dev. Biol. 8, 203 (2020).

4. M. Nobis, S. C. Warren, M. C. Lucas, K. J. Murphy, D. Herrmann, P. Timpson, Molecular mobility and activity in an intravital imaging setting-implications for cancer progression and targeting. J. Cell Sci. 131, jcs206995 (2018).

5. A. Coste, M. H. Oktay, J. S. Condeelis, D. Entenberg, Intravital imaging techniques for biomedical and clinical research. Cytometry A 97, 448-457 (2020).

6. M. Alieva, L. Ritsma, R. J. Giedt, R. Weissleder, J. van Rheenen, Imaging windows for long-term intravital imaging: General overview and technical insights. Intravita/ 3 , e29917 (2014).

7. A. Holtmaat, T. Bonhoeffer, D. K. Chow, J. Chuckowree, V. De Paola, S. B. Hofer, M. Hübener, T. Keck, G. Knott, W.-C. A. Lee, R. Mostany, T. D. Mrsic-Flogel, E. Nedivi, C. Portera-Cailliau, K. Svoboda, J. T. Trachtenberg, L. Wilbrecht, Long-term, high-resolution imaging in the mouse neocortex through a chronic cranial window. Nat. Protoc. 4, 1128-1144 (2009).

8. H. A. Lehr, M. Leunig, M. D. Menger, D. Nolte, K. Messmer, Dorsal skinfold chamber technique for intravital microscopy in nude mice. Am. J. Pathol. 143, 1055-1062 (1993).

9. G. M. Palmer, A. N. Fontanella, S. Shan, G. Hanna, G. Zhang, C. L. Fraser, M. W. Dewhirst, In vivo optical molecular imaging and analysis in mice using dorsal window chamber models applied to hypoxia, vasculature and fluorescent reporters. Nat. Protoc. 6, 1355-1366 (2011).

10. D. Entenberg, S. Voiculescu, P. Guo, L. Borriello, Y. Wang, G. S. Karagiannis, J. Jones, F. Baccay, M. Oktay, J. Condeelis, A permanent window for the murine lung enables high-resolution imaging of cancer metastasis. Nat. Methods 15, 73-80 (2018).

11. D. Kedrin, B. Gligorijevic, J. Wyckoff, V. V. Verkhusha, J. Condeelis, J. E. Segall, J. van Rheenen, Intravital imaging of metastatic behavior through a mammary imaging window. Nat. Methods 5, 1019-1021 (2008).

12. A. Zomer, S. I. J. Ellenbroek, L. Ritsma, E. Beerling, N. Vrisekoop, J. Van Rheenen, Intravital imaging of cancer stem cell plasticity in mammary tumors. Stem Cells 31, 602-606 (2013).

13. L. Ritsma, E. J. A. Steller, S. I. J. Ellenbroek, O. Kranenburg, I. H. M. B. Rinkes, J. van Rheenen, Surgical implantation of an abdominal imaging window for intravital microscopy. Nat. Protoc. 8, 583-594 (2013).

14. L. Ritsma, E. J. A. Steller, E. Beerling, C. J. M. Loomans, A. Zomer, C. Gerlach, N. Vrisekoop, D. Seinstra, L. van Gurp, R. Schäfer, D. A. Raats, A. de Graaff, T. N. Schumacher, E. J. P. de Koning, I. H. B. Rinkes, O. Kranenburg, J. van Rheenen, Intravital microscopy through an abdominal imaging window reveals a pre-micrometastasis stage during liver metastasis. Sci. Transl. Med. 4, 158ra145 (2012).

15. N. Rakhilin, A. Garrett, C.-Y. Eom, K. R. Chavez, D. M. Small, A. R. Daniel, M. M. Kaelberer, M. A. Mejooli, Q. Huang, S. Ding, D. G. Kirsch, D. V. Bohórquez, N. Nishimura, B. B. Barth, X. Shen, An intravital window to image the colon in real time. Nat. Commun. 10, 5647 (2019).

16. N. Hansen-Algenstaedt, C. Schaefer, L. Wolfram, C. Joscheck, M. Schroeder, P. Algenstaedt, W. Rüther, Femur window-A new approach to microcirculation of living bone in situ. J. Orthop. Res. 23, 1073-1082 (2005).

17. Q. Huang, M. A. Cohen, F. C. Alsina, G. Devlin, A. Garrett, J. McKey, P. Havlik, N. Rakhilin, E. Wang, K. Xiang, P. Mathews, L. Wang, C. Bock, V. Ruthig, Y. Wang, M. Negrete, C. W. Wong, P. K. L. Murthy, S. Zhang, A. R. Daniel, D. G. Kirsch, Y. Kang, B. Capel, A. Asokan, D. L. Silver, R. Jaenisch, X. Shen, Intravital imaging of mouse embryos. Science 368, 181-186 (2020).

18. C. Heo, H. Park, Y.-T. Kim, E. Baeg, Y. H. Kim, S.-G. Kim, M. Suh, A soft, transparent, freely accessible cranial window for chronic imaging and electrophysiology. Sci. Rep. 6, 27818 (2016). 
19. S. S. Cha, M. E. Bucklin, X. Han, Removable cranial window for sustained wide-field optical imaging in mouse neocortex. bioRxiv, 2020.01.14.905851 (2020).

20. L. Ghanbari, R. E. Carter, M. L. Rynes, J. Dominguez, G. Chen, A. Naik, J. Hu, M. A. K. Sagar, L. Haltom, N. Mossazghi, M. M. Gray, S. L. West, K. W. Eliceiri, T. J. Ebner, S. B. Kodandaramaiah, Cortex-wide neural interfacing via transparent polymer skulls. Nat. Commun. 10, 1500 (2019).

21. T. Sobolik, Y.-J. Su, W. Ashby, D. K. Schaffer, S. Wells, J. P. Wikswo, A. Zijlstra, A. Richmond, Development of novel murine mammary imaging windows to examine wound healing effects on leukocyte trafficking in mammary tumors with intravital imaging. Intravital $\mathbf{5}$, e1125562 (2016).

22. F. Helmchen, W. Denk, Deep tissue two-photon microscopy. Nat. Methods 2, 932-940 (2005)

23. K. W. Dunn, P. A. Young, Principles of multiphoton microscopy. Nephron Exp. Nephrol. 103, e33-e40 (2006).

24. M. D. Muzumdar, B. Tasic, K. Miyamichi, L. Li, L. Luo, A global double-fluorescent Cre reporter mouse. Genesis 45, 593-605 (2007).

25. B. C. Schaefer, M. L. Schaefer, J. W. Kappler, P. Marrack, R. M. Kedl, Observation of antigen-dependent CD8 ${ }^{+}$T-cell/dendritic cell interactions in vivo. Cell. Immunol. 214, 110-122 (2001).

26. Z. Hao, K. Rajewsky, Homeostasis of peripheral B cells in the absence of B cell influx from the bone marrow. J. Exp. Med. 194, 1151-1164 (2001).

27. S. Takaoka, Y. Kamioka, K. Takakura, A. Baba, H. Shime, T. Seya, M. Matsuda, Live imaging of transforming growth factor- $\beta$ activated kinase 1 activation in Lewis lung carcinoma $3 \mathrm{LL}$ cells implanted into syngeneic mice and treated with polyinosinic:polycytidylic acid. Cancer Sci. 107, 644-652 (2016).

28. J. Lau, C. C. Goh, S. Devi, J. Keeble, P. See, F. Ginhoux, L. G. Ng, Intravital multiphoton imaging of mouse tibialis anterior muscle. Intravital 5, e1156272 (2016).

29. M. T. Webster, U. Manor, J. Lippincott-Schwartz, C.-M. Fan, Intravital imaging reveals ghost fibers as architectural units guiding myogenic progenitors during regeneration. Cell Stem Cell 18, 243-252 (2016).

30. M. M. Murphy, J. A. Lawson, S. J. Mathew, D. A. Hutcheson, G. Kardon, Satellite cells, connective tissue fibroblasts and their interactions are crucial for muscle regeneration. Development 138, 3625-3637 (2011).

31. B. Evano, S. Khalilian, G. Le Carrou, G. Almouzni, S. Tajbakhsh, Dynamics of asymmetric and symmetric divisions of muscle stem cells in vivo and on artificial niches. Cell Rep. $\mathbf{3 0}$ 3195-3206.e7 (2020).

32. M.-E. Fernandez-Sanchez, T. Brunet, J.-C. Röper, E. Farge, Mechanotransduction's impact on animal development, evolution, and tumorigenesis. Annu. Rev. Cell Dev. Biol. 31, 373-397 (2015).

33. C. Matthews, F. P. Cordelières, in ImageJ User \& Developer Conference (2010).

34. H. Wickham, ggplot2 (Springer, 2009), pp. 1-7.

35. A. J. Ewald, Z. Werb, M. Egeblad, Monitoring of vital signs for long-term survival of mice under anesthesia. Cold Spring Harb. Protoc. 2011, pdb.prot5563 (2011).

36. A. Parslow, A. Cardona, R. J. Bryson-Richardson, Sample drift correction following 4D confocal time-lapse Imaging. J. Vis. Exp., e51086 (2014).

37. E. Stewart, S. M. Federico, X. Chen, A. A. Shelat, C. Bradley, B. Gordon, A. Karlstrom, N. R. Twarog, M. R. Clay, A. Bahrami, B. B. Freeman III, B. Xu, X. Zhou, J. Wu, V. Honnell,
M. Ocarz, K. Blankenship, J. Dapper, E. R. Mardis, R. K. Wilson, J. Downing, J. Zhang, J. Easton, A. Pappo, M. A. Dyer, Orthotopic patient-derived xenografts of paediatric solid tumours. Nature 549, 96-100 (2017).

Acknowledgments: We acknowledge P. Jacquemin (Lycée Marcellin Berthelot, Questembert-plastic engineering section) for help on the window and mold design and initial prototyping and for teaching us the basics in polymer properties and processing. We thank L. Sengmanivong, M. Irondelle, and O. Leroy from the Cell and Tissue Imaging (PICT-IBiSA) facility and the Nikon Imaging Centre, Institut Curie, member of the French National Research Infrastructure France-Biolmaging (ANR10-INBS-04). We are also grateful to R. Margueron (Institut Curie) for advice and constructive discussions. We acknowledge O. Delattre and S. Zaidi (Institut Curie) for providing the PDX tumors, in addition to the Institut Curie In Vivo Experimental Facility for help in the maintenance and care of the mouse colony. Funding: This work was supported by grants to S.F., B.L.-L., and G.J. from Paris Sciences et Lettres University (PSL Research University), the Institut Carnot, the French National Research Agency (ANR, grant no. ANR-15-CE13-0013-01), the Cancéropôle Île-de-France (grant no. 2015-2-APD-01-ICR-1), the Ligue contre le cancer (grant no. RS19/75-101), the LABEX DEEP ANR, number 11-LBX-0044, and by the "FRM Equipes" EQU201903007821 and the FSER (La Fondation Schlumberger pour I'Education et la Recherche) FSER20200211117. The PICT-IBiSA facility is supported by the Fondation pour la Recherche Médicale (FRM no. DGE20111123020), the Cancéropôle Île-de-France (no. 2012-2-EML-04-IC-1), and the INCA (Cancer National Institute, no. 2011-1-LABEL-IC-4). D.S. and A.B. are supported by the Institut Curie SIRIC (Site de Recherche Intégrée en Cancérologie, INCa-DGOS-Inserm_12554; ITMO Cancer AVIESAN). B.L.-L. acknowledges support from the Academy of Medical Sciences, Wellcome Trust, and the University of Bristol. S.T. acknowledges funding support from the Institut Pasteur, Agence Nationale de la Recherche (Laboratoire d'Excellence Revive, Investissement d'Avenir; ANR-10-LABX-73), M.B.-D. was supported by the Laboratoire d'Excellence Revive and La Ligue Contre le cancer. Author contributions: G.J., S.F., and B.L.-L. conceived and administered the study. G.J., M.B.-D., V.D.-M., D.S., S.T., S.F., and B.L.-L. conceived and designed the experiments. G.J., M.B.-D., S.D., A.B., and B.L.-L. performed the experiments. G.J., S.F., and B.L.-L., wrote the manuscript. All authors reviewed and approved the manuscript. Competing interests: The PDMS imaging window described in this publication is patented under the number EP3656349A1 by Institut Curie, where G.J., B.L.-L., and S.F. are named inventors. All other authors declare that they have no competing interests. Data and materials availability: All data needed to evaluate the conclusions in the paper are present in the paper and/or the Supplementary Materials. Additional data related to this paper may be requested from the authors

Submitted 27 January 2021

Accepted 30 April 2021

Published 16 June 2021

$10.1126 /$ sciadv.abg7663

Citation: G. Jacquemin, M. Benavente-Diaz, S. Djaber, A. Bore, V. Dangles-Marie, D. Surdez, S. Tajbakhsh, S. Fre, B. Lloyd-Lewis, Longitudinal high-resolution imaging through a flexible intravital imaging window. Sci. Adv. 7, eabg7663 (2021). 


\section{ScienceAdvances}

\section{Longitudinal high-resolution imaging through a flexible intravital imaging window}

Guillaume Jacquemin, Maria Benavente-Diaz, Samir Djaber, Aurélien Bore, Virginie Dangles-Marie, Didier Surdez, Shahragim Tajbakhsh, Silvia Fre and Bethan Lloyd-Lewis

Sci Adv 7 (25), eabg7663.

DOI: $10.1126 /$ sciadv.abg7663

ARTICLE TOOLS

SUPPLEMENTARY MATERIALS

REFERENCES

PERMISSIONS http://advances.sciencemag.org/content/7/25/eabg7663

http://advances.sciencemag.org/content/suppl/2021/06/14/7.25.eabg7663.DC1

This article cites 33 articles, 7 of which you can access for free http://advances.sciencemag.org/content/7/25/eabg7663\#BIBL

http://www.sciencemag.org/help/reprints-and-permissions

Science Advances (ISSN 2375-2548) is published by the American Association for the Advancement of Science, 1200 New York Avenue NW, Washington, DC 20005. The title Science Advances is a registered trademark of AAAS.

Copyright @ 2021 The Authors, some rights reserved; exclusive licensee American Association for the Advancement of Science. No claim to original U.S. Government Works. Distributed under a Creative Commons Attribution License 4.0 (CC $\mathrm{BY})$. 